\title{
Date of Death
}

National Cancer Institute

\section{Source}

National Cancer Institute. Date of Death. NCI Thesaurus. Code C70810.

The calendar date of subject's death. 